Published in final edited form as:

Virus Res. 2011 December ; 162(1-2): 8-11. doi:10.1016/j.virusres.2011.09.035.

\title{
Taming influenza viruses
}

Makoto Ozawa ${ }^{a, b,{ }^{*}}$ and Yoshihiro Kawaokaa,b,c,d, ${ }^{a}$

aDepartment of Special Pathogens, International Research Center for Infectious Diseases, Institute of Medical Science, University of Tokyo, Shirokanedai, Minato-ku, Tokyo, Japan

${ }^{b}$ Department of Pathobiological Sciences, School of Veterinary Medicine, University of WisconsinMadison, Madison, WI, USA

CInternational Research Center for Infectious Diseases, Institute of Medical Science, University of Tokyo, Shirokanedai, Minato-ku, Tokyo, Japan

dERATO Infection-Induced Host Responses Project, Japan Science and Technology Agency, Saitama, Japan

\section{Abstract \\ Plasmid-based reverse genetics systems allow the artificial generation of viruses with cloned cDNA-derived genomes. Since the establishment of such systems for influenza virus, numerous attempts have been made to tame this pathogenic agent. In particular, several types of viruses expressing foreign genes have been generated and used to further our knowledge of influenza virus replication and pathogenicity and to develop novel influenza vaccines. Here, we review these achievements and discuss future perspectives.}

\section{Keywords}

Influenza virus; reverse genetics; foreign gene expression

\section{Introduction}

In the last century, we experienced three influenza A virus pandemics: Spanish influenza in 1918, Asian influenza in 1957, and Hong Kong influenza in 1968. The Spanish influenza virus alone killed 20-50 million people worldwide (Wright et al., 2007). These events, together with annual influenza epidemics, have compelled us to develop effective measures to control influenza virus infection.

The genome of influenza A viruses, which are members of the Orthomyxoviridae family, comprises eight segmented negative-strand RNAs (PB2, PB1, PA, HA, NP, NA, M, and NS gene segments) that encode at least 11 viral proteins (Palese and Shaw, 2007). Each viral RNA (vRNA) consists of a 3'-end non-coding sequence, an internal coding sequence, and a

(C) 2011 Elsevier B.V. All rights reserved

*Corresponding authors. Mailing address: Makoto Ozawa Influenza Research Institute, Department of Pathobiological Sciences, School of Veterinary Medicine, University of Wisconsin-Madison, 575 Science Drive, Madison, WI 53711. Phone: (608) 890-1526 Fax: (608) 890-2912 mozawa@ vetmed.wisc.edu. “Yoshihiro Kawaoka Influenza Research Institute, Department of Pathobiological Sciences, School of Veterinary Medicine, University of Wisconsin-Madison, 575 Science Drive, Madison, WI 53711. Phone: (608) 265-4925 Fax: (608) 262-5622 kawaokay@svm.vetmed.wisc.edu.

Publisher's Disclaimer: This is a PDF file of an unedited manuscript that has been accepted for publication. As a service to our customers we are providing this early version of the manuscript. The manuscript will undergo copyediting, typesetting, and review of the resulting proof before it is published in its final citable form. Please note that during the production process errors may be discovered which could affect the content, and all legal disclaimers that apply to the journal pertain. 
5'-end non-coding sequence: 12 - and 13 -terminal nucleotides at the 3 ' and 5' ends, respectively, are highly conserved among the eight vRNAs (Fig. 1). Influenza virus is unique among RNA viruses in that its vRNAs are transcribed and replicated in the nucleus not in cytoplasm of infected cells.

Advances in molecular biological technologies have enabled the development and improvement of prophylactic and therapeutic interventions against influenza. In particular, since we (Neumann et al., 1999) and others (Fodor et al., 1999) established plasmid-based reverse genetics systems, a molecular technique for the artificial generation of viruses with cloned cDNA-derived genomes, for influenza viruses, our knowledge about influenza have been greatly extended and countermeasures against influenza have been dramatically improved. Indeed, this DNA engineering-based technique is now used in the preparation of live attenuated vaccines against seasonal and highly pathogenic H5N1 influenza viruses (Karron et al., 2009; Subbarao and Katz, 2004).

Such molecular techniques also open the door to a new field of influenza virology: creating foreign gene-expressing viruses that include not only influenza, but also some well-studied viruses such as retroviruses, lentiviruses, and adenoviruses. These 'tamed' influenza viruses have been used to gain new insights into influenza virus replication and pathogenicity and to develop new types of influenza vaccines. Moreover, because influenza viruses do not produce DNA intermediates in their replication cycle and are potent immune response stimulators, influenza viral vectors are considered to be a promising vaccine platform. Here, we summarize the efforts that have been made to generate influenza A viruses that express foreign genes and discuss the application of such studies.

\section{Influenza viruses expressing a foreign gene}

The first influenza virus harboring a foreign gene was reported by Luytjes et al. (Luytjes et al., 1989). They synthesized a virus-like RNA that encoded a reporter gene flanked by the 3' and 5' non-coding sequences of the NS vRNA by means of T7 polymerase-mediated in vitro transcription and transfected cultured cells with the RNA construct along with purified viral polymerase subunits (PB2, PB1, and PA) and nucleoprotein NP. Helper virus infection prior to the transfection ensured that the recombinant virus-like RNA was packaged into progeny viruses and expressed in cells that were subsequently infected with these viruses. These results indicate that both ends of the non-coding sequences serve as key elements in the transcription, replication, and incorporation of a foreign gene-encoding vRNA. Further, these findings also accelerated the engineering of influenza viruses harboring genes of interest (Enami et al., 1990).

Neumann et al. (Neumann et al., 1994) established a plasmid transfection-based system to express influenza virus-like RNAs. They cloned the cDNA of the virus-like RNA between the promoter and terminator sequences of RNA polymerase I (PolI), which localizes in nucleoli and normally catalyzes ribosomal RNA transcription. The virus-like RNA was expressed in the nuclei by transfection with the gene cassette-encoding plasmid and then transcribed and replicated by subsequent infection with helper viruses. This alternative approach eliminated several cumbersome processes, such as viral protein purification and in vitro RNA transcription; however, the need for helper virus infection to rescue the foreign gene-harboring viruses from the vast background of helper viruses restricted the application of this technique to viruses that could be picked up by selective pressure.

Helper virus-free systems for influenza virus generation were subsequently developed by Neumann et al. (Neumann et al., 1999) and Fodor et al. (Fodor et al., 1999). In place of helper virus infection, the eight vRNAs were provided from plasmids possessing the cDNAs of the viral genes under the control of the PolI promoter and the PolI terminator (Neumann 
et al., 1999) or the hepatitis delta virus-derived ribozyme (Fodor et al., 1999). In both systems, protein expression plasmids for the viral polymerase subunits and NP were transfected into cells to support initial transcription and replication of the vRNAs. These modifications extended the range of applications for this influenza virus engineering technique: theoretically, influenza viruses possessing any foreign genes could now be generated.

One of the most critical insights for the generation of foreign gene-expressing influenza viruses was obtained by Fujii et al. (Fujii et al., 2003) who found that in addition to noncoding sequences, partial coding sequences at both the 3 ' and 5' ends of the NA vRNA contribute to the efficient and selective packaging of the NA vRNA into virions. This concept was then demonstrated to be true for the remaining seven gene segments (Dos Santos Afonso et al., 2005; Fujii et al., 2005; Fujii et al., 2009; Gog et al., 2007; Liang et al., 2005; Liang et al., 2008; Marsh et al., 2007; Marsh et al., 2008; Muramoto et al., 2006; Ozawa et al., 2007; Ozawa et al., 2009; Watanabe et al., 2003) (Fig. 1). These findings allowed the design of recombinant influenza vRNAs suitable for efficient and stable incorporation into virions.

\section{Replication-competent vs. -incompetent viruses}

To date, several types of foreign gene-expressing influenza viruses have been created. These viruses are categorized into two groups based on their self-replication ability: replicationcompetent and -incompetent viruses. Both types of virus have strengths and limitations (Table 1). The replication-competent foreign gene-expressing viruses are an attractive experimental tool to investigate virus replication, especially in vivo, although their risk of revertant generation and potential pathogenicity can cause biosafety concerns. By contrast, their biologically contained replication-incompetent counterparts have fewer biosafety issues and the potential to accelerate in vitro studies, although they usually require special growth conditions to compensate for their replication-incompetency and allow their efficient replication.

\subsection{Replication-competent viruses}

Kittel et al. (Kittel et al., 2004) and Shinya et al. (Shinya et al., 2004) generated replicationcompetent influenza viruses possessing the green fluorescent protein (GFP) gene in the NS and NA vRNAs, respectively. Both viruses replicated efficiently under modified cell culture conditions; however, their replication was severely hampered under normal culture condition because a portion of the authentic coding sequences of these viruses was replaced with the GFP gene. Therefore, their replication kinetics and in vivo tropism did not necessarily reflect those of authentic viruses, although the NA-deficient GFP-expressing viruses were used to detect neutralizing antibodies against H5-subtype viruses (Rimmelzwaan et al., 2011).

Manicassamy et al. generated replication-competent GFP-expressing viruses of greater potency by using the porcine teschovirus-1-derived $2 \mathrm{~A}$ protease cleavage site to express the NS1-GFP fusion protein and 'almost' authentic NS2 protein from a single recombinant NS vRNA (Manicassamy et al., 2010). This cloning strategy allowed the recombinant virus to express a full-set of functional viral proteins, and the recombinant virus grew reasonably in normal cell culture. Moreover, this GFP-expressing virus was lethal to mice, although its pathogenicity was 100 -fold lower than that of authentic virus. These results highlight the potential applications of replication-competent foreign gene-expressing influenza viruses for the in vivo study of influenza virus pathogenicity. Li et al. (Li et al., 2010) also generated replication-competent viruses that expressed the GFP gene from the recombinant NA vRNA. Although they also preserved the authentic NA coding sequence and inserted the 
GFP gene as an 'extra gene element' along with a 2A peptide sequence (origin unknown), in vivo growth kinetics or pathogenicity of their viruses was not reported.

Other virus-derived glycoproteins have also been accommodated in replication-competent recombinant influenza viruses. Watanabe et al. (Watanabe et al., 2003) cloned the vesicular stomatitis virus glycoprotein (VSVG) gene into the HA vRNA. Since VSVG protein could functionally substitute for influenza viral envelope proteins, (i.e., HA and NA), the NA gene segment was open for insertion of an additional foreign gene. These authors therefore chose to generate recombinant influenza viruses possessing the VSVG and GFP genes in the HA and NA vRNAs, respectively, and demonstrated the stability of the inserted genes during serial virus passages. To compensate for the lack of NA function, Maeda et al. (Maeda et al., 2005) replaced the ectodomain of NA with that of Sendai virus-derived hemagglutininneuraminidase $(\mathrm{HN})$ protein. The resultant NA-HN chimeric protein not only supported NAdeficient virus replication but also generated a recombinant virus that simultaneously displayed influenza viral (HA) and Sendai viral (HN) major protective antigens on virions and infected cells. The bivalent vaccine potency of this replication-competent virus was validated in a mouse model, although its potential pathogenicity was observed (Maeda et al., 2005).

\subsection{Replication-incompetent viruses}

Since most of influenza viral proteins are indispensable for virus replication, single geneknockouts render influenza viruses replication-incompetent. Marsh et al. (Marsh et al., 2007) established a cell line stably expressing HA protein and demonstrated that this cell line supported efficient replication of viruses that possessed either the GFP or the red fluorescent protein (RFP) gene in their HA vRNA. These authors did not, however, report on the replicative ability of their HA-knockout (HA-KO) reporter gene-possessing viruses in normal cell culture. Martínez-Sobrido et al. (Martinez-Sobrido et al., 2010) followed up this study by establishing cell lines expressing HAs derived from various virus strains, including highly pathogenic H5-subtype and 'Spanish influenza' pandemic viruses. They showed that their HA-KO GFP-expressing virus was replication-incompetent without in trans HA expression. Although the stability of the reporter HA gene during virus replication remains unclear, this HA-KO system was demonstrated to be a safe and powerful tool to screen influenza viral HA-specific neutralizing antibodies.

While studying the mechanism underlying the spread of oseltamivir-resistant mutants, Bloom et al. developed a PB1-KO system (Bloom et al., 2010) and demonstrated that a virus possessing either the GFP or the mCherry gene in the PB1 vRNA replicated efficiently in cells expressing the PB1 protein. However, these replication-incompetent viruses were not characterized in detail in their study and several questions, such as the stability of the reporter PB1 gene during virus passages, remain unanswered.

Recently, we developed a PB2-KO system by replacing the viral PB2 gene with reporter genes and by establishing a PB2-expressing cell line (Ozawa et al., 2011). The growth kinetics of the PB2-KO viruses in the PB2-expressing cells was comparable to those of authentic virus, and no progeny viruses were recovered from normal cell culture. HA and/or NA genes could be replaced with other virus strain counterparts without preparing additional cell lines that expressed the viral proteins. More importantly, unlike the HA-KO system (Marsh et al., 2007), a seven-segmented virus lacking the PB2 vRNA did not undergo multiple replication cycles even in cells expressing the PB2 protein in trans, and thus the reporter gene-encoding PB2 vRNA was stably inherited in progeny viruses. We also demonstrated the potential of the PB2-KO system as a novel vaccine platform and obtained promising preliminary data in a mouse model (unpublished data). These results demonstrate 
the superior reliability and greater potential of the PB2-KO reporter gene-expressing viruses over other replication-incompetent influenza viruses.

\section{Future perspectives}

Despite abundant efforts to understand influenza virus pathogenicity, the mechanisms by which this pathogenic agent cause disease and kill animals and humans remain largely unclear. Replication-competent foreign gene-expressing influenza viruses are promising experimental tools to track virus-infected cell populations and to gain insights into pathogenicity. However, as exemplified by the replication-competent virus expressing the NS1-GFP fusion protein (Manicassamy et al., 2010), foreign gene insertion typically causes severe virus attenuation that obfuscates data interpretation. In addition, the stability of these foreign genes needs to be improved; Manicassamy et al. found that GFP-negative virus populations increased over time in cell culture, reaching $45 \%$ at $84 \mathrm{~h}$ post-infection. Accordingly, next-generation replication-competent influenza viruses will need to retain pathogenicity comparable to that of authentic viruses and to stably express foreign genes during multiple replication cycles.

Currently, cell-based high-throughput screening plays a critical role in identifying antiinfluenza viral compounds (Maddry et al., 2011) and cellular factors involved in influenza virus replication (Brass et al., 2009; Hao et al., 2008; Karlas et al., 2010; Konig et al., 2010). Since the equipment required for high-throughput screening is often built in biologically "uncontained" facilities, the availability of replication-incompetent foreign gene-expressing influenza viruses should be of benefit to these research areas. In fact, Hao et al. (Hao et al., 2008) and Konig et al. (Konig et al., 2010) applied replication-incompetent foreign geneexpressing influenza viruses to their genome-wide siRNA screenings.

Replication-incompetent viruses could also serve as vaccine candidates. Although these viruses do not produce progeny viruses under normal conditions, their vRNAs are transcribed and replicated in infected cells to some extent; for example, we detected significant levels of reporter gene expression even in PB2-KO virus-infected normal cell culture (Ozawa et al., 2011). These results indicate that replication-incompetent viruses have advantages over inactivated and live attenuated vaccines in terms of providing antigen and biosafety, respectively. Moreover, a foreign gene of interest can be accommodated as a secondary vaccine antigen derived from another pathogen, thereby generating bivalent vaccines. It may also be possible to combine multiple recombinant gene segments to prepare trivalent (or multi-valent) vaccines.

In conclusion, since the development of plasmid-based reverse genetics technologies, influenza viruses are no longer just pathogens, but also useful biologically engineered tools. Replication-competent and -incompetent influenza viruses that express foreign genes are likely to further our understanding of influenza virus pathogenicity and aid in the development of countermeasures against influenza viruses.

\section{Acknowledgments}

We thank Susan Watson for editing the manuscript. This work was supported, in part, by Grants-in-Aid for Specially Promoted Research and for Scientific Research, by a Contract Research Fund for the Program of Founding Research Centers for Emerging and Reemerging Infectious Diseases, by ERATO (Japan Science and Technology Agency), by the Special Coordination Funds for Promoting Science and Technology from the Ministry of Education, Culture, Sports, Science, and Technology of Japan, and by National Institute of Allergy and Infectious Diseases Public Health Service research grants, USA. 


\section{References}

Bloom JD, Gong LI, Baltimore D. Permissive secondary mutations enable the evolution of influenza oseltamivir resistance. Science. 2010; 328(5983):1272-1275. [PubMed: 20522774]

Brass AL, Huang IC, Benita Y, John SP, Krishnan MN, Feeley EM, Ryan BJ, Weyer JL, van der Weyden L, Fikrig E, Adams DJ, Xavier RJ, Farzan M, Elledge SJ. The IFITM proteins mediate cellular resistance to influenza A H1N1 virus, West Nile virus, and dengue virus. Cell. 2009; 139(7):1243-1254. [PubMed: 20064371]

Dos Santos Afonso E, Escriou N, Leclercq I, van der Werf S, Naffakh N. The generation of recombinant influenza A viruses expressing a PB2 fusion protein requires the conservation of a packaging signal overlapping the coding and noncoding regions at the 5' end of the PB2 segment. Virology. 2005; 341(1):34-46. [PubMed: 16084555]

Enami M, Luytjes W, Krystal M, Palese P. Introduction of site-specific mutations into the genome of influenza virus. Proc Natl Acad Sci U S A. 1990; 87(10):3802-3805. [PubMed: 2339122]

Fodor E, Devenish L, Engelhardt OG, Palese P, Brownlee GG, Garcia-Sastre A. Rescue of influenza A virus from recombinant DNA. J Virol. 1999; 73(11):9679-9682. [PubMed: 10516084]

Fujii K, Fujii Y, Noda T, Muramoto Y, Watanabe T, Takada A, Goto H, Horimoto T, Kawaoka Y. Importance of both the coding and the segment-specific noncoding regions of the influenza A virus NS segment for its efficient incorporation into virions. J Virol. 2005; 79(6):3766-3774. [PubMed: 15731270]

Kawaoka Y. Importance of both the coding and the segment-specific noncoding regions of the influenza A virus NS segment for its efficient incorporation into virions. J Virol. 2005; 79(6):37663774. [PubMed: 15731270]

Fujii K, Ozawa M, Iwatsuki-Horimoto K, Horimoto T, Kawaoka Y. Incorporation of influenza A virus genome segments does not absolutely require wild-type sequences. J Gen Virol. 2009; 90(Pt 7): 1734-1740. [PubMed: 19297607]

Fujii Y, Goto H, Watanabe T, Yoshida T, Kawaoka Y. Selective incorporation of influenza virus RNA segments into virions. Proc Natl Acad Sci U S A. 2003; 100(4):2002-2007. [PubMed: 12574509]

Gog JR, Afonso Edos S, Dalton RM, Leclercq I, Tiley L, Elton D, von Kirchbach JC, Naffakh N, Escriou N, Digard P. Codon conservation in the influenza A virus genome defines RNA packaging signals. Nucleic Acids Res. 2007; 35(6):1897-1907. [PubMed: 17332012]

Hao L, Sakurai A, Watanabe T, Sorensen E, Nidom CA, Newton MA, Ahlquist P, Kawaoka Y. Drosophila RNAi screen identifies host genes important for influenza virus replication. Nature. 2008; 454(7206):890-893. [PubMed: 18615016]

Karlas A, Machuy N, Shin Y, Pleissner KP, Artarini A, Heuer D, Becker D, Khalil H, Ogilvie LA, Hess S, Maurer AP, Muller E, Wolff T, Rudel T, Meyer TF. Genome-wide RNAi screen identifies human host factors crucial for influenza virus replication. Nature. 2010; 463(7282):818-822. [PubMed: 20081832]

Karron RA, Talaat K, Luke C, Callahan K, Thumar B, Dilorenzo S, McAuliffe J, Schappell E, Suguitan A, Mills K, Chen G, Lamirande E, Coelingh K, Jin H, Murphy BR, Kemble G, Subbarao $\mathrm{K}$. Evaluation of two live attenuated cold-adapted $\mathrm{H} 5 \mathrm{~N} 1$ influenza virus vaccines in healthy adults. Vaccine. 2009; 27(36):4953-4960. [PubMed: 19540952]

Kittel C, Sereinig S, Ferko B, Stasakova J, Romanova J, Wolkerstorfer A, Katinger H, Egorov A. Rescue of influenza virus expressing GFP from the NS1 reading frame. Virology. 2004; 324(1): 67-73. [PubMed: 15183054]

Konig R, Stertz S, Zhou Y, Inoue A, Hoffmann HH, Bhattacharyya S, Alamares JG, Tscherne DM, Ortigoza MB, Liang Y, Gao Q, Andrews SE, Bandyopadhyay S, De Jesus P, Tu BP, Pache L, Shih C, Orth A, Bonamy G, Miraglia L, Ideker T, Garcia-Sastre A, Young JA, Palese P, Shaw ML, Chanda SK. Human host factors required for influenza virus replication. Nature. 2010; 463(7282): 813-817. [PubMed: 20027183]

Li F, Feng L, Pan W, Dong Z, Li C, Sun C, Chen L. Generation of replication-competent recombinant influenza A viruses carrying a reporter gene harbored in the neuraminidase segment. J Virol. 2010; 84(22):12075-12081. [PubMed: 20826692] 
Liang Y, Hong Y, Parslow TG. cis-Acting Packaging Signals in the Influenza Virus PB1, PB2, and PA Genomic RNA Segments. J Virol. 2005; 79(16):10348-10355. [PubMed: 16051827]

Liang Y, Huang T, Ly H, Parslow TG, Liang Y. Mutational analyses of packaging signals in influenza virus PA, PB1, and PB2 genomic RNA segments. J Virol. 2008; 82(1):229-236. [PubMed: 17959657]

Luytjes W, Krystal M, Enami M, Pavin JD, Palese P. Amplification, expression, and packaging of foreign gene by influenza virus. Cell. 1989; 59(6):1107-1113. [PubMed: 2598262]

Maddry JA, Chen X, Jonsson CB, Ananthan S, Hobrath J, Smee DF, Noah JW, Noah D, Xu X, Jia F, Maddox C, Sosa MI, White EL, Severson WE. Discovery of novel benzoquinazolinones and thiazoloimidazoles, inhibitors of influenza H5N1 and H1N1 viruses, from a cell-based highthroughput screen. J Biomol Screen. 2011; 16(1):73-81. [PubMed: 21059874]

Maeda Y, Hatta M, Takada A, Watanabe T, Goto H, Neumann G, Kawaoka Y. Live bivalent vaccine for parainfluenza and influenza virus infections. J Virol. 2005; 79(11):6674-6679. [PubMed: 15890905]

Manicassamy B, Manicassamy S, Belicha-Villanueva A, Pisanelli G, Pulendran B, Garcia-Sastre A. Analysis of in vivo dynamics of influenza virus infection in mice using a GFP reporter virus. Proc Natl Acad Sci U S A. 2010; 107(25):11531-11536. [PubMed: 20534532]

Marsh GA, Hatami R, Palese P. Specific residues of the influenza A virus hemagglutinin viral RNA are important for efficient packaging into budding virions. J Virol. 2007; 81(18):9727-9736. [PubMed: 17634232]

Marsh GA, Rabadan R, Levine AJ, Palese P. Highly conserved regions of influenza a virus polymerase gene segments are critical for efficient viral RNA packaging. J Virol. 2008; 82(5):2295-2304. [PubMed: 18094182]

Martinez-Sobrido L, Cadagan R, Steel J, Basler CF, Palese P, Moran TM, Garcia-Sastre A. Hemagglutinin-pseudotyped green fluorescent protein-expressing influenza viruses for the detection of influenza virus neutralizing antibodies. J Virol. 2010; 84(4):2157-2163. [PubMed: 19939917]

Muramoto Y, Takada A, Fujii K, Noda T, Iwatsuki-Horimoto K, Watanabe S, Horimoto T, Kida H, Kawaoka Y. Hierarchy among Viral RNA (vRNA) Segments in Their Role in vRNA Incorporation into Influenza A Virions. J Virol. 2006; 80(5):2318-2325. [PubMed: 16474138]

Neumann G, Watanabe T, Ito H, Watanabe S, Goto H, Gao P, Hughes M, Perez DR, Donis R, Hoffmann E, Hobom G, Kawaoka Y. Generation of influenza A viruses entirely from cloned cDNAs. Proc Natl Acad Sci U S A. 1999; 96(16):9345-9350. [PubMed: 10430945]

Neumann G, Zobel A, Hobom G. RNA polymerase I-mediated expression of influenza viral RNA molecules. Virology. 1994; 202(1):477-479. [PubMed: 8009859]

Ozawa M, Fujii K, Muramoto Y, Yamada S, Yamayoshi S, Takada A, Goto H, Horimoto T, Kawaoka Y. Contributions of two nuclear localization signals of influenza A virus nucleoprotein to viral replication. J Virol. 2007; 81(1):30-41. [PubMed: 17050598]

Ozawa M, Maeda J, Iwatsuki-Horimoto K, Watanabe S, Goto H, Horimoto T, Kawaoka Y. Nucleotide sequence requirements at the 5' end of the influenza A virus M RNA segment for efficient virus replication. J Virol. 2009; 83(7):3384-3388. [PubMed: 19158245]

Ozawa M, Victor ST, Taft AS, Yamada S, Li C, Hatta M, Das SC, Takashita E, Kakugawa S, Maher EA, Neumann G, Kawaoka Y. Replication-incompetent influenza A viruses that stably express a foreign gene. J Gen Virol. 2011

Palese, P.; Shaw, ML. Orthomyxoviridae: the viruses and their replication. In: Knipe, DM.; Howley, PM., editors. Fields virology. 5th ed.. Lippincott-Raven Publishers; Philadelphia, Pa.: 2007. p. 1647-1689.

Rimmelzwaan GF, Joyce Verburgh R, Nieuwkoop NJ, Bestebroer TM, Fouchier RA, Osterhaus AD. Use of GFP-expressing influenza viruses for the detection of influenza virus A/H5N1 neutralizing antibodies. Vaccine. 2011; 29(18):3424-3430. [PubMed: 21396410]

Shinya K, Fujii Y, Ito H, Ito T, Kawaoka Y. Characterization of a neuraminidase-deficient influenza a virus as a potential gene delivery vector and a live vaccine. J Virol. 2004; 78(6):3083-3088. [PubMed: 14990727] 
Subbarao K, Katz JM. Influenza vaccines generated by reverse genetics. Curr Top Microbiol Immunol. 2004; 283:313-342. [PubMed: 15298174]

Watanabe T, Watanabe S, Noda T, Fujii Y, Kawaoka Y. Exploitation of nucleic acid packaging signals to generate a novel influenza virus-based vector stably expressing two foreign genes. J Virol. 2003; 77(19):10575-10583. [PubMed: 12970442]

Wright, PF.; Neumann, G.; Kawaoka, Y. Orthomyxoviruses. In: Knipe, DM.; Howley, PM., editors. Fields virology. 5th ed.. Lippincott-Raven Publishers; Philadelphia, Pa.: 2007. p. 1691-1740. 


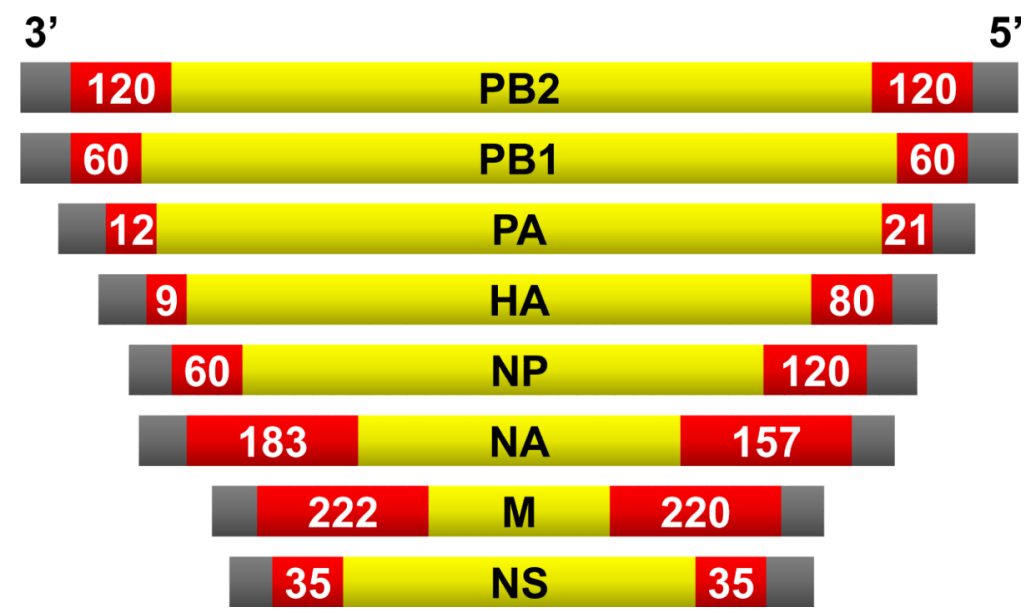

Fig. 1. Schematic diagram of the coding regions required for efficient genome packaging Noncoding and coding regions are represented by grey and yellow bars, respectively. Genespecific coding regions required for efficient genome packaging are represented by red bars. Numbers in the red bars indicate the number of nucleotides. 
Table 1

Key features of replication-competent and -incompetent viruses.

\begin{tabular}{|c|c|c|}
\hline & \multicolumn{2}{|c|}{ Specific feature of: } \\
\hline & Replication-competent viruses & Replication-incompetent viruses \\
\hline Strength & Mirror wild-type virus behavior & Limited biosafety concerns \\
\hline \multirow{2}{*}{ Limitation } & Risk of reversion to a wild-type sequence & Require special growth condition \\
\hline & Potential pathogenicity & Limited applications for in vivo study \\
\hline
\end{tabular}

